\section{Accidental Poisoning in Childhood}

SIR,-While welcoming the attention directed by Dr. R. H. Jackson and others (26 October, p. 245) and Dr. W. P. Sweetnam (16 November, p. 453) to the growing problem of accidental poisoning in childhood, I would like to correct a statement of fact (16 November, p. 453) that there were " 1,000 hospital beds in daily use."

The estimated number of beds in use during 1965 for cases of accidental poisoning were:

\begin{tabular}{c|c|c|c|c|c} 
Age: & $0-$ & $1-4$ & $5-9$ & $10-12$ & $13-14$ \\
\hline Males & 2 & 41 & 5 & 5 & 2 \\
Females & 4 & 40 & 2 & 2 & 5
\end{tabular}

These are the average number throughout the year, rounded to the nearest integer, and inciude the multiplying factor (which for the 1965 sample was 10.831). When numbers "included in the Enquiry" are quoted in the reports they need to be multiplied by 10 or more to obtain an estimate of the total number, but rates or other derived statistics already have this multiplying factor incorporated.-I am, etc.,

W. A. WILSON. Gcneral Register Office,
London W.C.2.

\section{Preventing Postoperative Thrombosis}

SIR,-In your leading article (10 August, p. 329) you stressed the high incidence of pulmonary embolism revealed by a special search of the lungs at necropsy, and also that many deaths from this condition may be " unnecessary." Anticoagulants have been used to prevent thrombosis and embolism in both medical and surgical patients with undoubted success, but with some complications; low molecular weight dextrans may also have some value in this respect. However, another simple and apparently harmless method of prevention may appeal to some surgeons.

Evidence is accumulating which suggests that thrombosis in the legs of surgical patients begins during the operation itself, while the leg muscles are flaccid and subject to compression from the operating-table; meanwhile changes in the flow and coagulability of blood, which are known to occur at this time, are likely to increase the risk of clotting. Doran and White ${ }^{1}$ have shown the incidence and severity of thromboembolic disease in high-risk surgical patients are considerably reduced by stimulating the calf muscles electrically during surgery. But deep vein thromboses are known to involve, in significant incidence, the veins in the thighs and the various iliac veins as well as those of the calf; furthermore, a search by one of us of the intramuscular and larger gluteal vessels in a small number of necropsies revealed antemortem thrombi, although the incidence of this manifestation is not known. We have therefore developed a simple method of stimulating the whole of both lower limbs during operations, and our preliminary findings in general surgical patients are consistent with those of Doran and White.

The apparatus required is in everyday use in almost all departments of physical medicine, and its operation is easily learnt. The stimulator must bc capable of delivering D.C. (galvanic) stimuli of up to $150 \mathrm{~A}$ to curarized patients, but surged A.C. (faradic) stimuli are adequate for all other patients: both forms of stimulation are provided by the Universette of similar apparatus. The electrodes consist of aluminium plates placed in thick lint bags wetted with weak saline solution, and these are connected by $7-\mathrm{ft}$. lengths of wire to the stimulator.

When the patient is anaesthetized a large proximal clectrode $(20 \mathrm{~cm}$. by $25 \mathrm{~cm}$.), supported by a half-full rubber (hot-water) bottle to improve electrical contact, is placed behind the lumbar lordosis and connected to one terminal of the stimulator; two small distal electrodes $(15 \mathrm{~cm}$. by $5 \mathrm{~cm}$.) are then secured to the popliteal fossae by means of Velcro straps, and both are connected to the other terminal. When the stimulator has warmed up the current is increased until good intermittent contractions of the glutei, the thigins, and the calves can be seen, but if the two legs do not move equally the tension of the Velcro straps need to be altered; the contractions are maintained until the operation ends. The arbitrary rate chosen for the stimuli is once every eight seconds. For operations on the legs, including varicose veins, the distal electrodes are placed behind the buttocks and the current adjusted to produce isometric contractions, but for operations in the lateral position and those on the back two small $(15 \mathrm{~cm}$. by $5 \mathrm{~cm}$.) proximal electrodes are used instead of one, and these are strapped on to the upper thighs behind the sciatic nerves.

Non-explosive anaesthesia is mandatory with the equipment currently available, and no metal must touch the patient's skin. Local erythema resulting from electrolysis of salt is avoided by washing the lint bags thoroughly between cases or by reversing the polarity of the electrodes every half an hour. The technique does not preclude the use of diathermy, the negative plate being applied to the upper part of the body. We prefer to elevate the legs by about 15 degrees by placing them on Sorbo rubber cushions which also increase the weight-bearing areas of the calves and thighs. -We are, etc.,

\section{G. E. MOLONEY.}

The Radcliffe Infirmary.

$$
\text { R. H. FELL. }
$$
\section{Oxford}

\section{REFERENCE}

Doran, F. S. A., and White, H. M., Brit. ? Surg., 1967, 54, 686.

\section{Puerperal Thromboembolism}

SIR,-I am interested in recent letters regarding the increased incidence of puerperal thrombosis and pulmonary embolism, blaming stilboestrol as a possibility (16 November, p. 451).

What about oxytocic drugs ? These cause a vascular spasm and are known to be dangerous in patients with toxaemia. In my considered opinion the routine use of oxytocic drugs by midwives in normal deliveries requires thought and reconsideration. Do we know the optimum normal blood loss? The larger the baby, the larger the placental site and normal loss. What is physiological ? A normal puerperal haemoglobin should be achieved.

Hurrying the third stage leads to an increased incidence of retained chorion and even placental tissue. Although primary haemorrhage undoubtedly is diminished by the use of oxytocic drugs, the incidence of secondary haemorrhage seems to have increased. The tendency now is to encourage midwives to interfere with nature, and pupil midwives seldom see a truly normal delivery. Doctors have created many iatrogenic diseases. Surely midwives, through the Central Midwives Board, should try to avoid this by adhering to nature, except in emergencies.-I am, etc.,

$$
\begin{aligned}
& \text { JEAN L. HALLUM. } \\
& \text { Sorrento Maternity Hospital, } \\
& \text { Birmingham 13. }
\end{aligned}
$$

\section{Management of Pulmonary Embolism}

SIR,-Over the past two years there have been three leading articles in the B.M.F. on the management of pulmonary embolism, the most recent on 19 October (p. 133). Common to all has been the assertion that embolectomy with cardiopulmonary bypass has a role in the management of patients with massive pulmonary embolism. I would like to suggest that this mode of therapy is by no means as well established as you would have us believe, and that many of the reasons used to justify the operation are based on false premises.

Thus thrombosis occurs distal to the region where the clot lodges in around $70 \%$ of subjects with pulmonary embolism. This secondary thrombosis extends well out into the peripheral vessels. The concept that all is well once the major embolus is removed is wishful thinking, as is the belief that retrograde " milking" enables the surgeon to remove secondary thrombi from the more peripheral branches of the pulmonary artery. You then go on to say the mortality from embolectomy is around $50 \%$, but quote no source for this figure. Over the past five years in the two teaching hospitals with which I have been associated the mortality in something over 25 cases treated by embolectomy and the bypass has been around $90 \%$. These results cannot be attributed to inexperience or poor surgical technique, and it is evident when one talks to one's more realistic colleagues from similar institutions that their experience has been equally disappointing. I do not doubt that some surgeons have better results, but I would venture to suggest that their success can be accounted by selection. If one is to operate on subjects who do not really need surgery, one is bound to have some survivals.

It has been shown that, in both dogs and humans, most emboli recanalize within two to four weeks. ${ }^{12}$ Since this is so, were it possible to tide the patient over the acute effects and to prevent further emboli, embolectomy would be superfluous. The use of agents to lyse the clot would seem to be a far more worthwhile approach, and indeed this has been shown to be so. ${ }^{3}$ With regard to the prevention of additional emboli, surely inferior vena cava ligation or plication should have received some comment.

It is human nature to report only one's successes. Consequently we all read numerous case reports in the literature of isolated subjects surviving pulmonary embolectomy. Where are the reports of one or two survivors out of 20 subjects or on 10 consecutive deaths following pulmonary embolectomy? I would suggest that the latter type of article is far more useful to us all, but where is the surgeon 